\title{
Confidences féminines et sorcellerie : une ethnologie des émotions en terrain sensible
}

\section{Clara Lemonnier}

\section{(QpenEdition \\ Journals}

Édition électronique

URL : http://journals.openedition.org/pa/474

DOI : $10.4000 /$ pa. 474

ISSN : 2273-0362

Éditeur

Université Lumière Lyon 2

Édition imprimée

ISBN : 1634-7706

ISSN : $1634-7706$

Référence électronique

Clara Lemonnier, «Confidences féminines et sorcellerie : une ethnologie des émotions en terrain

sensible », Parcours anthropologiques [En ligne], 11 | 2016, mis en ligne le 20 décembre 2016, consulté le 19 avril 2019. URL : http://journals.openedition.org/pa/474 ; DOI : 10.4000/pa.474

Parcours anthropologique 


\title{
Confidences féminines et sorcellerie : une ethnologie des émotions en terrain sensible
}

\author{
Clara Lemonnier
}

Université de Bordeaux

\section{INTRODUCTION}

Les soins dits non conventionnels ${ }^{1}$ rencontrent actuellement un engouement croissant au sein de la population française ${ }^{2}$ (Schmidt, 2006 ; Grisoni, 2011 ; Rossi et Cohen, 2011 ; Sakoyan, Musso, Mulot, 2011), malgré les interdictions légales d'exercice des praticiens et les représentations négatives dont ces derniers font encore souvent l'objet, accusés d'exploiter la crédulité et la souffrance humaines. Les travaux anthropologiques en terrains européens éclairent le recours croissant des individus à un pluralisme thérapeutique, soit qu'ils recherchent un traitement efficace à un problème que la biomédecine a laissé sans réponse satisfaisante à leur yeux, soit qu'ils aspirent à une prise en charge plus individualisée et à des interprétations des maux personnalisées, donnant du sens à leur infortune (Cant, Sharma, 1999 ; Durish-Gauthier, 2007, Cohen, Rossi et al, 2010 ; Lemonnier, 2016). La méfiance envers les effets iatrogènes de la biomédecine ainsi que le sentiment d'une " déshumanisation » des soins médicaux participent à renforcer l'attrait pour les soins non conventionnels, perçus comme moins nocifs et plus attentifs aux spécificités de chacun sur les plans tant physique que psychique, moral, émotionnel et spirituel (Gasharian, 2002 ; Bishop, 2004). Cet article examine l'importante place accordée à l'expression et à la gestion des émotions dans ces pratiques de soins dans le cas précis du pluralisme thérapeutique consacré aux «maux de femmes ». Cette catégorie opératoire regroupe l'ensemble des troubles, déséquilibres, mal-être ou maladies décrits comme spécifiquement féminins par la soixantaine de femmes rencontrées dans la presqu'île du Médoc, dans le cadre de ma thèse et de ma participation à une enquête

\footnotetext{
${ }^{1}$ La législation européenne qualifie ainsi les pratiques thérapeutiques exercées en dehors du corps biomédical (Robard, 2002). La législation française quant à elle, interdit l'exercice médical à toute personne non titulaire du diplôme universitaire de médecine depuis la loi de 1892, revue en 1945. Les pratiques paramédicales et l'accompagnement social sont également soumis à l'obtention de diplômes reconnus par l'État. Les praticiens qui exercent des soins en dehors de ce cadre sont considérés comme enfreignant la loi par la pratique illégale de la médecine, ce dont ils se défendent en expliquant généralement que leurs soins ne sont ni biomédicaux ni n'empiètent sur le suivi médical des individus.

2 On estime actuellement selon les sources, qu'entre $40 \%$ et $75 \%$ des français ont déjà eu recours au moins une fois dans leur vie à des soins non conventionnels (OMS, 2002 et 2013; Miviludes, 2012 ; CAMbrella, 2010).
} 
collective sur l'accès aux soins en santé reproductive ${ }^{3}$. Ces maux s'accompagnent souvent de souffrances morales, parfois très profondes selon la gravité du problème, l'intensité de la douleur, la propension du trouble à perturber le quotidien et les représentations sociales négatives dont ils peuvent faire l'objet.

Constatant le recours de plusieurs enquêtées à des soins non conventionnels en Médoc, je me suis demandée en quoi ces praticiens répondaient différemment aux besoins des usagères. Les entretiens réalisés avec une quinzaine de praticiens non conventionnels, ainsi que les expériences de soins auxquelles je me suis livrée (de même que ma directrice de thèse et des amies ${ }^{4}$ ) permettent d'envisager ces pratiques thérapeutiques comme des espaces où les souffrances morales peuvent être prises en charge, en valorisant notamment l'expression d'émotions douloureuses généralement gardées confidentielles.

Proposant des pistes de réflexions pour l'anthropologie des émotions, cet article entend d'abord réaffirmer que les souffrances liées aux maux de femmes font l'objet de normes de pudeur et de tabous, lesquels impliquent le voilement, voire le refoulement, de leurs affects. Ensuite, il s'agit de montrer que les contextes thérapeutiques non conventionnels peuvent se révéler être des espaces propices à leur dévoilement, dans la mesure où les conceptions étiologico-thérapeutiques reposent sur l'expression même des émotions négatives. Enfin, l'intérêt est porté sur les transformations de soi liées au travail émotionnel mis en œuvre par les femmes qui recourent, ponctuellement ou régulièrement, à un pluralisme thérapeutique.

Plusieurs travaux consacrés aux émotions soulignent néanmoins la difficulté posée par leur caractère intime et intérieur, limitant de fait l'observation ethnographique (Paperman, 1995; Rimé, 2005) à moins d'adopter une démarche de recherche telle que celle élaborée Jeanne FavretSaada dans son enquête sur la sorcellerie dans le bocage normand (1977). Pour la chercheuse, l'ethnologue n'a véritablement accès qu'à ses propres ressentis, qu'il doit interroger en tant que caractéristique des statuts « indigènes » qui lui sont localement attribués. En acceptant d'occuper ces statuts particuliers plutôt que s'imaginer ce qu'on vivrait à la place de l'autre, l'ethnologue se trouve "affecté » par les situations étudiées et peut ressentir des émotions qu'il n'éprouverait pas dans un autre contexte (Favret-Saada, 2009:156). Cette

\footnotetext{
${ }^{3}$ Ma thèse, soutenue en juin 2016, s'intitule Quêtes de soins au féminin: une ethnographie des "maux de femmes" et du pluralisme thérapeutique en Médoc. Elle a été financée via un contrat doctoral avec l'Université de Bordeaux (Ecole doctorale SP2) et co-dirigée par le Prof. Abel Kouvouama (Université de Pau et des Pays de l'Adour) et le Dr. Laurence Kotobi (Université de Bordeaux). Cette dernière a dirigé de 2010 à 2013 une étude socio-anthropologique financée par l'Iresp-Inserm (à laquelle j'ai contribué entre 2011 et 2013) et portant sur les "inégalités dans l'accès aux soins en santé génésique et reproductive : le cas des femmes 'précaires et/ou migrantes' du Médoc », dans le département français de la Gironde.

${ }^{4}$ Celles-ci résidaient à Bordeaux ou dans la campagne alentour et étaient à ce moment-là respectivement étudiante, travailleuse sociale et musicienne.
} 
démarche semble particulièrement adaptée à l'étude des pratiques de soins non conventionnels qui ne répondent pas aux conceptions étiologicothérapeutiques de la biomédecine et qui nécessitent d'être abordées selon des méthodologies différentes ${ }^{5}$.

Ainsi, cet article mêle des réflexions tirées de la thèse et des considérations d'ordre plus réflexif quant aux statuts de confidente, d'usagère de soins non conventionnels et d'apprentie-sorcière que j'ai occupés pendant cette enquête. Des séquences ethnographiques de certaines de mes "relations instituantes » (Rossi, 2002) sont restituées dans le détail de leur déroulement et donnent à voir les échanges particuliers qui ont eu lieu selon que j'occupe telle ou telle place: il s'agit des confidences de notre interlocutrice privilégiée ici appelée Christine, des séances et des entretiens menés avec l'une de ses thérapeutes nommée Laura, et de mon début d'initiation à ce qu'elles considèrent comme de la sorcellerie. Si ce récit peut paraitre anecdotique dans sa forme, il a l'avantage de porter l'attention sur les émotions ressenties, leur traitement social spécifique dans ces contextes et les changements que cela a suscité dans ma posture en tant qu'anthropologue et en tant que jeune femme. Ces aspects apparaissent utiles à la compréhension des mécanismes à l'œuvre dans certains soins non conventionnels, lesquels semblent favoriser l'expression des affects des patientes, participer d'un travail émotionnel allégeant les souffrances, tout en menant à la redéfinition de soi en tant que femme, dans les relations aux autres et dans les rapports de genre.

\section{LES SOUFFRANCES DE FEMMES : UNE ETHNOGRAPHIE SENSIBLE}

\section{Une parole marquée par la pudeur et le tabou}

La recherche collective menée dans le Médoc (Kotobi et al, 2013) a beaucoup influencé mon enquête sur le pluralisme thérapeutique dans ce territoire rural du Sud-Ouest de la France. L'hétérogénéité des lieux ethnographiés et des personnes enquêtées me l'ont fait connaitre au-delà des clichés habituels, qui opposent les vins prestigieux et les plages océanes à la mauvaise réputation des médocains, souvent décrits à Bordeaux comme archaïques, alcooliques et consanguins. Accéder à des réalités moins stéréotypées a demandé de prendre

\footnotetext{
${ }^{5}$ L'anthropologue Ilario Rossi insiste sur l'intérêt de considérer les relations établies sur le terrain comme des «relations instituantes", à partir desquelles s'effectue son travail de réflexivité, tant pour le recueil des données que pour leur analyse, ce qui lui a permis de saisir toute la place de la parole dans les soins chamaniques chez les Huichols du Mexique (Rossi, 2002). Deirdre Meintel adopte elle aussi une démarche immersive et réflexive dans ses travaux sur les médiums au Québec. Au travers de sa propre expérience initiatique et de ses relations avec les initiés et les adeptes de l'Eglise où elle a enquêté, elle éprouve les logiques d'apprentissage de cette pratique dite surnaturelle, qu'elle découvre caractérisée par un certain lâcher-prise et une écoute attentive et régulière de ses propres ressentis émotionnels, ensuite verbalisés auprès d'un public en attente de révélations.
} 
le temps de nouer de vraies relations et de se faire connaitre sur le terrain, et pas seulement en tant qu'étudiante bordelaise et citadine. Je devais montrer que j'étais une jeune femme digne de confiance, originaire de la proche côte landaise et familière de certains codes sociaux en usage sur la côte médocaine, comme le sentiment d'attachement au territoire ou la revendication plus ou moins utilitariste d'une identité culturelle. Le Médoc m'est apparu comme un lieu de contrastes, faisant coexister de richissimes châtelains avec des ouvriers viticoles invisibilisés, souvent précaires. Réputé pour la quiétude de ces espaces naturels et un supposé art de vivre sauvage, il attire nombre de néoruraux et des milliers de touristes. Les habitants revendiquent avec fierté un mode de vie insulaire tout en se plaignant de leur enclavement par rapport à Bordeaux. Espace aux frontières administratives floues, il est marqué par une certaine rareté médicale en ce qui concerne notamment les soins spécialisés dans la santé des femmes.

Mes rencontres avec celles-ci se sont d'abord organisées grâce à des amies étudiantes bordelaises, travaillant l'été dans les stations balnéaires. Elles se sont ensuite déroulées en différents lieux d'enquête, certains étant communs à la recherche collective - centres de planification familiale, services d'accompagnement social, salle d'attente de médecins, associations, politique locale $\mathrm{d}^{\prime}$ action sanitaire...- et $\mathrm{d}^{\prime}$ autres plus spécifiques à mon thème de recherche (stages de développement personnel, cours collectifs de yoga, soins aux domiciles et cabinets de thérapeutes non conventionnels). Les premières enquêtées nous présentant à leurs proches, j'ai pu rencontrer une soixantaine de femmes, âgées de 16 à 76 ans, originaires ou non de la presqu'île et y résidant au moins une partie de l'année. Leurs profils regroupaient des lycéennes, des étudiantes, des employées à l'année ou "à la saison », des femmes sans emplois ou des mères au foyer (un à sept enfants). Elles étaient célibataires, mariées, pacsées, séparées ou divorcées et une minorité avait des préférences homosexuelles. Toutes relataient des parcours en santé reproductive aussi variés que singuliers.

Lors de nos entretiens, je constatai qu'il n'était pas toujours aisé de recueillir le vécu personnel des maux de femmes. "Avez-vous des problèmes de femmes ? » avais-je l'habitude de leur demander, tout en observant que les façons dont elles me disaient ou me taisaient leurs réponses étaient liées à la qualité de la confiance préalablement établie ainsi qu'à l'intimité et l'anonymat offert par le contexte de l'entretien. Cette règle instituant la pudeur dans le dévoilement de soi en tant que femme $\mathrm{m}^{\prime}$ avait aussi été enseigné petite fille: les maux de femmes ne se racontent pas en public et nécessitent toujours un espace de confiance et de confidence, souvent féminin, pour être exprimés, au risque de susciter en public une réaction de rejet, voire de dégoût (Douglas, 1971 ; Mardon, 2011). Une culture de la dissimulation est transmise aux jeunes filles, la transgression des normes pouvant les stigmatiser comme incapables de maitriser leur sexualité ou leur fécondité. 
Comme moi, chacune des membres de l'équipe avait personnellement fait l'objet d'un apprentissage des normes de pudeur et de tabou relatives aux corps féminins, lesquelles resurgissaient parfois en miroir dans les situations d'enquête. Travaillant sur un terrain proche et sur des questions nous concernant parfois personnellement et intimement, il n'a pas été rare pour de se sentir éprouvées par les entretiens, surtout quand ils faisaient écho à nos propres expériences. Intégrer ces vécus au travail ethnographique et à la réflexion permet de constater qu'à l'instar des femmes rencontrées, le caractère secret ou tabou de nos maux semblait plus ou moins prononcé selon le problème, ses représentations, l'âge, le statut socio-professionnel, l'origine sociale et culturelle et les expériences traversées en santé reproductive. La retenue de chacune à parler de ses expériences s'est peu à peu amenuisée au fil du temps, laissant place à une forme d'entre-soi féminin. Il en est allé de même avec les femmes enquêtées qui ont accepté de nous parler de leurs expériences, souvent à l'issue de plusieurs rencontres, et dans des contextes favorisant la confidence, comme l'espace privé de leur maison, de leur voiture, ou un lieu extérieur (parc, café) perçu comme neutre et anonyme. Elles ont parfois écarté les questions qu'elles trouvaient trop indiscrètes, ou trop douloureuses, quand ce n'était pas nous qui craignions de susciter une gêne. Quand ces difficultés étaient enfin dépassées, tant du côté des enquêtrices que des enquêtées, ces dernières exprimaient alors parfois des tristesses, des peurs, des colères, des résignations. La récurrence de ces réactions émotionnelles $\mathrm{m}^{\prime}$ ont amenée à penser qu'elles étaient le fruit des tensions entre un vécu intérieur douloureux et l'impossibilité normée et normative de pouvoir l'exprimer.

Taire ou confier un évènement ou une situation ayant perturbé l'équilibre émotionnel et affectif, résulte d'un " travail émotionnel » par lequel s'établit le choix d'exprimer (ou de cacher) ses émotions conformément ou non à la connaissance que l'on a des contextes socio-culturels dans lesquels on vit et des modes d'affiliation qui y ont cours. Le vécu intime des émotions n'est donc pas extérieur aux «savoirs affectifs » véhiculés par la culture du groupe auquel on appartient, car même si chacun inscrit l'émotion "plutôt à la première personne », c'est toujours "au sein d'un tissu de significations et d'attitudes qui imprègne simultanément les manières de la dire et de la mettre physiquement en jeu » (Le Breton, 2004 : 4). Le jeu prescrit de voilement et de dévoilement des émotions est toujours dépendant des contextes sociaux et des rapports de pouvoir qui s'y jouent (Fernandez, Lézé, Marche, 2008 : 4). Marcel Mauss pointait déjà ces enjeux de maintien des liens d'appartenance et de cohésion sociale qui se posent aux membres d'un groupe, lesquels doivent manifester leurs émotions aux moments, selon des attitudes et selon une intensité socialement appropriés (Mauss, 1921). Cela génère parfois des tensions entre le vécu intime des émotions et leur manifestation auprès d'autrui, qui se doit d'être socialement compréhensible et acceptable (Paperman, 1995). Cette tension était particulièrement palpable dans les 
silences ou les discours d'évitements recueillis auprès des femmes rencontrées dans le Médoc. Elles paraissaient contraintes (comme nous aussi parfois) de dissimuler au quotidien leurs souffrances.

Les normes de pudeur participent ainsi à masquer les souffrances morales de femmes, qui acceptent de vivre avec des affections des parties génitales ou des douleurs de règles par habitude ou par crainte de la stigmatisation. Les troubles dépressifs liés à l'infertilité, à des problèmes d'ordre sexuel ou à une forme d'anormalité physique (l'hermaphrodisme ou l'ablation d'un sein suite à un cancer) ne bénéficient pas toujours d'une forme de reconnaissance sociale permettant une compréhension des troubles et des prises en charge adaptées, et ce sans doute davantage dans les déserts médicaux. Cette invisibilisation est d'autant plus problématique que les souffrances liées à des problèmes considérés comme graves demeurent néanmoins peu dicibles, comme les traumatismes laissés par des expériences d'IVG, de violences conjugales ou de viols. Ces ressentis parfois profonds de mal-être influencent en retour le pouvoir de dire, de se raconter et de s'estimer (Ricoeur, 1994), agissant sur la capacité de se réaliser en tant que femme. En effet, les normes fixant socialement les limites de l'exposition de soi chez les femmes sont à replacer dans la perspective structurelle plus large de la valence différentielle des sexes, tel que développée par Françoise Héritier (1996). L'apprentissage et l'intériorisation des règles de pudeur et de tabou participent au maintien renouvelé de rapports de genre inégaux à un niveau macro-social.

\section{Le récit de vie d'une femme : Christine}

La restitution de ma relation ethnographique avec Christine donne à voir en contexte, le récit qu'elle nous a livré concernant le vécu de maux généralement tenus au secret. En même temps elle montre la place de l'ethnologue dans ce type d'enquête, enjoint à établir de forts liens de confiance dans la durée mais aussi à entendre des récits de vie parfois difficiles auxquels il n'est pas toujours préparé.

J'ai fait la connaissance de Christine par le biais de Laurence Kotobi, ma directrice de thèse. Lors de leur rencontre sur un marché du Médoc où elle vendait des produits locaux, Christine s'était montrée volontaire pour me rencontrer dans le cadre de ma recherche. En effet, les deux femmes s'étaient mises à discuter d'un livre que lisait alors Christine et qui traitait d'une technique thérapeutique hawaïenne. L'anthropologue apprit ce jour-là qu'elle était atteinte d'une maladie orpheline contre laquelle elle se battait avec l'aide de médecins, de psychologues mais aussi de thérapeutes aux pratiques non conventionnelles. Un premier rendez-vous fut alors fixé et je la rencontrai chez elle quelques semaines plus tard.

Lors de ce premier rendez-vous, Christine nous a invitées à déjeuner chez elle. A notre arrivée, elle nous a chaleureusement proposé de la suivre. A l'entrée de la maison, trois grosses pierres. Christine nous a expliqué qu'il s'agissait d'obsidiennes ayant la particularité de pouvoir " empêcher les 
personnes néfastes d'entrer ». Etant ainsi autorisées, nous nous sommes assises dans le salon. Un climat de confiance avait été établi précédemment entre Laurence Kotobi et cette femme qui, informée de notre méthodologie par entretien, a accepté sans problème que nous utilisions un dictaphone alors que nous lui demandions de raconter son « histoire en tant que femme ».

Là a commencé son récit auquel on ne se s'attendait pas: née à l'étranger d'une famille française dans les années 50 , elle avait vécu la plus grande partie de sa vie dans sa petite ville médocaine. Elle a expliqué avoir " toujours été en souffrance " : son père, " autoritaire et sévère », a abusé d'elle durant son enfance, lui laissant de lourds traumatismes psychiques et physiques que Christine tente de réparer courageusement chaque jour. Pour échapper à cette violence familiale, elle s'est mariée jeune pour emménager dans un foyer plus sécurisant. Hélas, son mari s'est révélé-également violent sexuellement, l'obligeant à des pratiques qu'elle refusait. Cet homme exerçait une profession à risque qui a détérioré sa santé et notamment ses fonctions reproductives. Ce pourquoi, elle n'a pas eu d'enfant avec lui. Son deuxième mari ne voulait pas d'enfants. De toute façon, elle a découvert à cette époque qu'elle ne pouvait pas donner la vie : "C'est à cause de ce que m'a fait mon père... Mes ovaires se sont collés contre la paroi et se sont atrophiés. "Ce deuxième mari est décédé prématurément. Christine nous a alors raconté avoir vécu par la suite deux grandes histoires d'amour, puis le quotidien du célibat, parfois lourd pour quelqu'un qui aime donner et recevoir du temps et de l'affection.

\section{Le rôle de la confidente}

Lorsque Christine nous raconta son histoire, le jour de notre rencontre, je me souviens être restée sous le choc après son récit détaillé des violences physiques et sexuelles qu'elle avait endurées. Des cauchemars en ont hanté mes nuits à plusieurs reprises. Admirative de son attitude toujours positive, j'ai rapidement eu beaucoup de respect pour Christine, en dépit de certaines rumeurs qui la disaient aussi gentille que bizarre. Cette réputation pouvait être confortée par le fait qu'elle se présentait parfois comme guérisseuse, ce qui pouvait susciter de la méfiance dans ce territoire où il est de bon ton de cacher ce type de croyances.

Comme ce fut aussi le cas pour d'autres femmes, j'ai rapidement compris que nous faisions partie des rares personnes à qui elle a raconté cette histoire si difficile et personnelle. Pendant la durée de l'entretien, j'ai d'abord tenté d'adopter, sans doute pour me rassurer, une posture proche de celle que j'avais observée chez les conseillères conjugales du service hospitalier d'orthogénie où j'avais enquêté auparavant (Lemonnier, 2010). Ces professionnelles de la santé reproductive et de la vie sexuelle et affective adoptaient une attitude distanciée face à la souffrance d'autrui, un regard empathique, une oreille attentive. Les travaux menés sur la gestion émotionnelle chez les professionnels de santé soulignent la maîtrise valorisée de leurs propres affects, construite comme un signe de leur compétence ou 
comme une protection contre la contagion symbolique ${ }^{6}$; ils encouragent parallèlement les patients au discours sur soi pour obtenir les indications cliniques dont ils ont besoin, tant en les incitant à contrôler des émotions qui perturberaient trop l'organisation du travail (Drulhe, 2000, 2006 ; Mercadier, 2002 ; Memmi, 2004). J'ai régulièrement discuté de la posture à tenir avec ma directrice de thèse -qui elle s'appuyait sur une posture entre distance, conseil et empathie, apprise lors de sa formation d'infirmière et son expérience d'anthropologue- lors des débriefings en équipe ou de discussions informelles. Une transmission du métier a pu s'effectuer ainsi parfois à mon insu, sur «comment être à l'écoute », «amener vers » ou encore "savoir répondre » lorsqu'on est soit même affectée par le sujet abordé.

La posture envisagée au départ de ma relation avec Christine n'était pas vraiment adaptée et allait rapidement évoluer au gré de la relation. J'abandonnai peu à peu mon effort de maîtrise émotionnelle que j'avais dû fournir sur mon précédent terrain hospitalier, pour envisager une posture plus symétrique et égalitaire, que ce soit avec elle ou avec les autres enquêtées.

Mon apprentissage de l'écoute et ma compassion pour les femmes, motivés par l'envie de mettre en lumière des situations ordinairement masquées, m'ont permises d'être intégrée auprès de mes interlocutrices en tant que confidente. Considérée comme une «étrangère proche » (Gallenga, 2010), j'avais l'avantage d'être une dépositaire de secrets peu susceptible d'enfreindre les règles de confidentialité dans un petit monde rural où « tout se sait». A l'inverse, il était aussi attendu de moi que je livre des secrets personnels, la confidence devant être réciproque. Je pense que cette posture, se distançant des modèles professionnels et modelée par mon engagement relationnel sur le terrain, a été décisive quant au recueil des récits de vie des femmes. En effet, la distance émotionnelle dans le travail des rares professionnels de la santé sexuelle et reproductive rencontrés en Médoc, ainsi que le ciblage de leurs missions (pour certaines pathologies et certaines populations «à risque »), peuvent contribuer à alimenter les normes de pudeur (en étant trop direct ou en ne laissant pas la place aux récits subjectifs par exemple), ce qui ne facilite pas l'expression du vécu des femmes. Il advient qu'un engagement réciproque dans la confidence et la manifestation d'une sincère compassion auprès des enquêtées a été nécessaire, pour accéder et comprendre leurs représentations des maux de femmes et leurs influences sur le vécu intime et les décisions thérapeutiques mises en œuvre.

Les travaux de sciences sociales portant sur la santé sexuelle et reproductive des femmes n'adoptent que rarement une approche réflexive de l'expérience de terrain et du vécu émotionnel des chercheuses, majoritaires dans ce domaine d'étude. L'empreinte militante des gender studies à l'origine de ce

6 « Il/Elle est trop dans l'affect » est une expression que j'ai souvent entendu dans le milieu professionnel médical, paramédical et médico-social pour signifier la trop forte empathie d'un collègue avec les bénéficiaires de ses soins et son manque de retenue/distance émotionnelle, exprimant implicitement un manque de professionnalisme de sa part. 
champ s'exprime le plus souvent dans des analyses critiques du biopouvoir caractéristiques de l'anthropologie de la santé, souvent au Nord centrée sur les mesures de santé publique et les prises en charge biomédicales. Si ces travaux témoignent souvent en filigrane de l'engagement politique et social des auteures (Browner, Sargent, 2011), leurs spécificités et ce qu'elles dévoilent de leur identité sont des précisions plus rares (Kotobi, 2013). Or, il apparait ici que la restitution et l'examen explicites des affects qui traversent les ethnologues sur les terrains proches sont pertinents pour rendre compte de la sensibilité des questions abordées et du positionnement qu'ils sont alors amenés à adopter, en fonction des règles sociales régissant les interactions et les possibilités ou non de les transgresser.

\section{L'INTERPRETATION DES EMOTIONS DANS LES SOINS NON CONVENTIONNELS}

Lorsque les femmes rencontrées n'ont pas pu ou n'ont pas souhaité exprimer leur problème auprès des professionnels de santé, ou qu'elles ont considéré qu'il n'était pas du ressort de l'institution médicale, certaines d'entre elles expliquaient s'être tournées vers d'autres voies thérapeutiques. La dimension psychologique et mystique des soins non conventionnels a déjà été soulignée (Rocchi, 2000 ; Bishop, 2004) et peut expliquer le recours de ces femmes à un pluralisme thérapeutique. Je me suis intéressée aux mécanismes individuels et sociaux à l'œuvre dans ce type de soins, encore peu étudiés par l'anthropologie au Nord (Favret, Contreras, 1990 ; Raineau, 2011), pour comprendre à un niveau phénoménologique, ce qui se joue pendant ces recours, en quoi ils permettent l'expression de maux parfois tabous et comment ils en modifient le vécu. Dans ma thèse, les récits des itinéraires thérapeutiques des usagères $\mathrm{m}$ 'ont permis $\mathrm{d}$ 'envisager ces recours comme des espaces permettant le dévoilement de ressentis habituellement masqués. J'ai souhaité ici interroger ces phénomènes au travers de ma propre expérience de recours à ce type de soins, ce que Christine (parmi d'autres) a rendu possible.

\section{Une consultation à haute charge émotionnelle}

Lors de ma première rencontre avec Christine, celle-ci nous a précisé que les effets handicapants de sa maladie l'avaient conduite à changer d'activité. Elle employait désormais son temps à l'invention de procédés écologiques ainsi qu'à une quête spirituelle tournée vers la guérison et la compréhension de grandes questions existentielles. Bien que suivie par son médecin traitant, par des spécialistes, une kinésithérapeute et une psychologue, Christine nous confia souffrir souvent physiquement et moralement. Après avoir consulté une dizaine de magnétiseurs dans la région, elle a rencontré Laura, une médium magnétiseuse à qui elle conférait un grand pouvoir soignant quant au maintien de sa mobilité corporelle et de son moral. 
Suite à une journée de rencontre qu'elle a organisée chez elle pour que nous échangions avec ses amies (j'y reviendrais plus loin), Christine nous a incitées à rencontrer l'une de ses thérapeutes, «parce que elle, elle fait tout ! C'est la crème. Pour la recherche, c'est elle qu'il vous faut ! ». Ce type de mise en relation s'est révélé des plus efficaces pour rencontrer des thérapeutes non conventionnels dans le Médoc, aux activités peu visibles car non autorisées en France. Suivant les analyses et les conseils de l'anthropologue Clémentine Raineau7, j'ai suivi «la rumeur» pour me faire introduire auprès des thérapeutes, méthode qui implique l'engagement du chercheur dans des relations de proximité et de longue durée avec ses interlocuteurs. Ainsi circulaient les informations renseignant la réputation et l'efficacité d'un praticien et avisant des suspicions de charlatanisme. Le bouche-à-oreille influence ainsi les choix thérapeutiques de la personne en quête de soins et du groupe qui l'accompagne ${ }^{8}$.

Selon Christine, notre rencontre avec sa thérapeute allait être autant utile à mon enquête qu'à notre bien-être. Nous interrogeant sur nos défaillances physiques ou morales à l'occasion de moments de confidences, elle était persuadée que Laura pouvait nous soulager. Elle proposa que nous organisions une nouvelle journée chez elle, au cours de laquelle nous pourrions consulter Laura chacune notre tour, moyennant 50 euros la séance d'une heure, puis réaliser un entretien enregistré avec elle. Je compris plus tard que Christine souhaitait aider Laura à lancer son auto-entreprise en «thérapies alternatives ». Laura l'avait remerciée par des tarifs préférentiels, contente d'avoir de nouvelles clientes, qui plus est chercheures en santé. Laurence Kotobi et moi-même sommes donc revenues chez Christine pour bénéficier d'une consultation thérapeutique peu ordinaire. Contrairement à ma directrice, j'avais déjà bénéficié de divers soins énergétiques suite à un évènement marquant de ma vie de femme. Pour autant, chaque thérapeute précédemment rencontré proposait des prises en charge particulières, me laissant moi aussi dans l'expectative de ce que nous allions vivre. Mes rencontres ultérieures avec divers praticiens confirmèrent qu'il est vain de vouloir dresser une typologie de ces thérapeutes - qui se disent guérisseurs, magnétiseurs, aromathérapeutes, praticien énergétique - tant leurs profils et activités sont hétérogènes. Leurs activités combinent des savoirs alliant bon sens populaire, empathie et prières tenues comme secrètes ${ }^{9}$, des savoirs professionnels, tirés de formations dans le

\footnotetext{
7 Clémentine Raineau, avec qui j'ai fait plusieurs entretiens, a réalisé une thèse sur les guérisseurs en Combrailles auvergnates en 2001 et s'est beaucoup intéressée à la " rumeur », c'est-à-dire au bouche-à-oreille qui colporte les expériences individuelles et les conseils d'orientations en matière de santé.

8 Clémentine Raineau (2001) explique aussi que donner une indication sur un thérapeute revient à rendre service à l'autre et à signifier son empathie envers lui. Ce dernier se trouve alors en dette et devra lui rendre service en retour ou plus simplement lui relater son expérience, laquelle alimentera la « rumeur ».

9 Certains praticiens pensent que les « dévoiler » leur ferait perdre leur pouvoir de guérison, c'est pourquoi lors des séances, ces prières sont marmonnées de façon inaudible.
} 
milieu paramédical et/ou dans les sphères alternatives, ainsi que des savoirs tirés de leurs propres expériences de maladie et de guérison.

Laura était arrivée la veille chez Christine car elle n'habitait pas le Médoc. Elles avaient réalisé ensemble un rituel de "ré-énergétisation des pierres à la pleine lune», nous laissant comprendre qu'elles partageaient des pratiques ésotériques. C'était une belle femme de 40 ans environ, ancienne intermittente $\mathrm{du}$ spectacle qui exerçait disait-elle, en tant que "sorcière moderne et décomplexée ». Laurence Kotobi fut la première conviée à sa consultation, dans une pièce calme de la maison. A son retour dans le salon, une heure plus tard, elle m'a fait signe de la tête pour me signifier qu'elle se sentait bien. Puis Laura lui a demandé de se tenir debout au milieu de la pièce afin de lui prodiguer un "grattage», grattant littéralement son dos puis semblant jeter dans l'air des résidus invisibles. Devant nos airs surpris, elle a précisé enlever les charges négatives qui pesaient comme un fardeau sur le dos de l'anthropologue.

Ça a été ensuite mon tour de bénéficier d'une consultation individualisée avec la "gentille sorcière ». Procédant d'abord à la lecture du thème astrologique qu'elle avait préparé (nous avions donné à Christine nos noms, prénoms et date de naissance à sa demande), elle a poursuivi par un tirage de cartes illustrées de personnages mythiques, complété par un tirage de runes10. Ces divers éléments ont constitué le support d'une discussion très dense et rythmée ; pour Laura, tout indiquait que je bénéficiais de "grandes capacités d'innovation, de créativité et d'intuition ", des qualités plutôt féminines selon elle que je devais laisser se développer, en acceptant de lâcher prise sur mes modes de pensée plus rationnalisants, vus comme masculins. Prise de doutes quant à ma capacité à aller au bout de ma thèse, j'ai accueilli ces propos comme de sincères encouragements. Je devais apprendre à me faire confiance et accepter "mon incarnation en tant que femme", et en tant que "petite sorcière » (j'y reviendrai), d'après ce qu'elle disait entendre des messages envoyés par "mes anges », appelés à venir grâce au son vibrant de son bol tibétain. Convaincue que mon incarnation dans cette vie avait pour dessein de me faire éprouver mon "côté Yin " et ma féminité (ce que confirmait son pendule), elle chercha à connaitre dans mon passé des épisodes difficiles ayant marqué des ruptures dans ma construction identitaire en tant que femme,

Généralement, ces prières sont révélées par le guérisseur aux seules personnes à qui il souhaite transmettre le pouvoir ou le don de guérison quand, devenu trop vieux ou fatigué, il décide d'arrêter cette activité.

10 Il s'agissait de choisir au hasard trois petits galets plats parmi plusieurs, sur l'envers desquels étaient inscrits des symboles que j'identifiais plus tard comme étant les lettres de l'alphabet runique. Cet alphabet était utilisé à l'Antiquité et au Moyen-Age dans les régions de l'actuelle Scandinavie comme système d'écriture et dans les arts divinatoires. Dans les sphères alternatives et ésotériques actuelles, les runes sont principalement utilisées dans les procédés de divination. Dans mon cas, les trois symboles figurant sur les trois galets choisis formaient une combinaison qui renvoyait à une grille d'interprétation, révélant alors le message que « l'univers » et « mes anges » devaient me transmettre par le biais de Laura. 
délogeant de façon très fine des souvenirs refoulés et des émotions contenues que je ne souhaite pas relater ici, par pudeur là encore. Christine lui avait surement explicité l'objet de nos enquêtes, ce qui l'avait peut-être aiguillée sur des questions qui me tenaient à cœur. Toujours est-il que ses questions et ses affirmations tombaient souvent juste au point que je me suis mise à pleurer. Laura m'a encouragé à " laisser les larmes venir » en tirant un mouchoir d'une boite visiblement prévue à cet effet. Elle m'a ensuite fait répéter des phrases où il était question de se pardonner à soi-même, comme aux autres, de n'avoir pas toujours été dans la bienveillance envers moi-même et de (se) remercier d'avoir su traverser ces expériences en tirant les enseignements que me destinait "l'univers» pour me guider dans " mon chemin de vie ». Elle m'a enfin donné des élixirs à boire chaque jour et des sels aux huiles essentielles pour mon appartement, «traitement purificatoire» que j'ai accepté, tout comme certains produits et objets magiques offerts par Christine. Des « rituels de protection » $m^{\prime}$ ont aussi été confiés spécifiquement pour me prémunir « du mal » que d'autres «guérisseurs » pourraient me causer au cours de mon enquête.

\section{Le dévoilement des émotions comme support de la thérapie}

Passés les premiers instants de fascination, j'ai perçu la consultation comme un moment hors du temps et de la vie ordinaire, favorisant le dévoilement de ressentis et d'émotions non socialement exprimables en dehors de ce contexte.

Laura procédait en gardant parfois la parole et en déclamant des affirmations d'ordre intime que je trouvais souvent justes à mon égard sans que je ne me sois auparavant sentie capable ou autorisée à les dire et les assumer ${ }^{11}$. A d'autres moments, elle instaurait un climat propice à ce que j'exprime de simples associations de mots ou la description parcellaire et désordonnée d'images ou de ressentis ${ }^{12}$, auxquels elle disait trouver du sens : «Mais c'est évident!» ou «Tout s'explique!». Le lâcher-prise encouragé à titre thérapeutique a eu un effet de catharsis qui ne pouvait se produire que dans le cadre précis de l'interaction avec la praticienne. Pour cette dernière, mes émotions ont constitué des indices précieux pour l'interprétation de mes «blocages» et de mes «croyances limitantes» ainsi qu'elle nommait les représentations de soi et $\mathrm{du}$ monde qui génèrent en chacun des peurs, des angoisses ou des sentiments d'impuissance, sur lesquelles elle proposait d'agir.

Elle appuyait sa pratique sur des référentiels médicaux et spirituels variés, et sur une conception endogène de l'étiologie des maux: les émotions

\footnotetext{
${ }^{11}$ Voir à ce propos, l'ouvrage Médecins et sorciers des ethnopsychiatres Tobie Nathan et Isabelle Stengers (1995) qui montrent que le « sorcier » prend la parole, conjure, ordonne et interprète, contrairement au psychanalyste qui cherche à faire parler son patient dans son processus thérapeutique.

12 Voir à ce propos le travail d'Ilario Rossi sur «la parole comme soin » (2011) et l'ouvrage dirigé par Rémi Bordes (2011) sur la place de la parole dans les médecines anciennes et contemporaines de par le monde.
} 
négatives étaient perçues comme responsables de dérèglements «sur le plan énergétique » causant du mal-être et maladies. Les savoirs et techniques mobilisées s'inscrivaient dans le courant New Age, mettant « l'accent sur une vision holiste de la personne - le corps, l'âme et l'esprit sont inter-reliés-, la faculté d'auto-guérison du corps, le pouvoir du psychisme sur les organes, la prévention des maladies par la quête de bien-être, la circulation des 'énergies' » (Durish-Gauthier, 2007 : 41). La notion d' "énergie » y est à la fois centrale et polysémique: elle peut se définir au prisme de différents référentiels comme les traditions savantes (les médecines humorale, chinoise et védique) ou certaines représentations des sciences physiques, en radiesthésie ou bioénergétique par exemple. Ces approches considèrent le corps et l'esprit dans un rapport intime capable d'influer sur la santé et organisent la thérapie sur la base de l'expression des émotions des patients pour situer le mal-être, avant d'en interpréter la signification et de mettre en œuvre un double travail $\mathrm{d}^{\prime}$ acceptation et de mise à distance du problème. Pour Christine par exemple, les différents soins réalisés par Laura permettaient de soulager ses «problèmes de femmes » évoqués plus haut, en agissant notamment sur le plan émotionnel et spirituel, pour favoriser son bien-être sur les plans affectif et physique.

\section{DE L'APPROPRIATION DU TRAVAIL EMOTIONNEL AU SOUCI DE SOI}

La consultation avec Laura, complétée par d'autres entretiens et observations sur le terrain, permet d'appréhender le dévoilement des émotions comme une nécessité étiologique et thérapeutique dans ce type de soins. Cette séance a également été un moment important de ma relation avec Christine et Laura, car elles m'ont poussée depuis lors à expérimenter d'autres façons d'être au monde, en apprenant notamment à incarner un rôle de sorcière. J'ai interprété cette invitation à l'initiation sorcellaire comme constituante $\mathrm{du}$ cheminement thérapeutique qu'elles mettaient en œuvre à mon égard, mais aussi comme une possibilité offerte $d^{\prime}$ appréhender par moimême le parcours dans lequel elles s'étaient engagées. Au-delà de l'expérience personnelle, cette expérience de terrain montre que le recours aux soins non conventionnels implique une sorte de transformation du regard porté sur soi et sur ses problèmes ${ }^{13}$. Cette transformation peut être ponctuelle et factuelle, ce qui aurait pu être le cas si je m’étais tenue à la seule séance de Laura. Elle peut aussi être entretenue par des recours réguliers aux praticiens non conventionnels puis par l'apprentissage de pratiques dites de « développement personnel » et de techniques magiques et mystiques, ce à quoi je décidais de me livrer.

13 Clémentine Raineau parle de "transformation » quant à la manière "d'être-au-monde » (2011 : s. p). 
Mon expérience de terrain permet de comprendre que cette «transformation de soi » est assez intérieure et passe d'abord par le dévoilement, le lâcher-prise et la prise de conscience des ressentis douloureux. S'ensuit un travail de réflexivité, c'est-à-dire de mise à distance de ses émotions pour mieux les comprendre dans leur contexte, menant à considérer les épisodes difficiles comme autant d'expériences riches de sens. Enfin, donner du sens à l'expérience nécessite d'interpréter les maux à la lumière de différents référentiels thérapeutiques, psychologiques, mystiques ou encore mythologiques, choisis par les thérapeutes pour leur propension à revaloriser le vécu des personnes. La quête de sens s'accompagne d'une quête de soi, dans la mesure où les référentiels choisis sont généralement porteurs de nouveaux repères identitaires valorisants auxquels on peut choisir de se conformer. Dans mon cas, la figure de référence choisie fut celle de la sorcière.

\section{Apprendre à être une sorcière}

Lors de ma première rencontre avec Christine, celle-ci avait précisé que Laura l'aidait à développer ses capacités extrasensorielles, elle qui disait avoir hérité des dons de guérison de sa grand-mère pour "panser le feu ». Croyante, elle avait « lu la Bible trois fois et le Coran deux fois ! ». Ce jour-là, alors qu'elle nous proposait de nous présenter des proches pour l'enquête, elle avait pris soin de vérifier à l'aide de son pendule si elle « faisait bien de les contacter». Assise " le dos au Nord », Christine laissa pendre l'objet à qui elle parla comme à un ami : "Bonjour! Tu vois, nous avons de la visite ! Ces jolies dames sont anthropologues et nous avons du boulot à faire. Veux-tu me donner un oui s'il te plaît ? »J'ai été charmée par la situation. J'échangeai quelques regards avec ma directrice qui semblait elle aussi surprise de la scène. Christine passa en revue et à voix haute chacune des personnes précédemment listées et le pendule oscilla différemment à deux reprises, ce qui a été interprété comme un conseil de ne pas contacter les deux personnes correspondantes.

Plus tard, lorsque nous sommes revenues le jour de la consultation avec Laura et que j'attendais mon tour, Christine a fait des allusions assez inhabituelles à mon sujet. " Tu sais que tu as quelque chose ? Tu es encore jeune... Mais va falloir commencer à canaliser tout ça! Holalala ! Et tu ne te protèges pas assez ! » Je compris qu'elle m'expliquait que j'avais des capacités magnétiques ou quelque chose d'approchant. Je lui ai demandé ce qu'elle voulait dire par là. "Que tu as quelque chose ! " répondit-elle fermement, puis énigmatique, «tu verras bien ce que te dit Laura tout à l'heure. ». Je lui ai alors confié que mon arrière-arrière-grand-père était guérisseur. Il vivait dans un petit village en Normandie et était connu pour enlever le feu. Je ne l'ai pas connu, mais mon père a gardé ses prières de guérison qu'il m'a transmises. Elle réagit placidement : "Tu vois, il n'y a pas de hasard. ». Bien qu'étonnée de cette révélation, je l'étais moins quant au choix d'enquêter sur les thérapeutes non conventionnels, mon histoire familiale $\mathrm{m}^{\prime}$ ayant souvent 
conduite à me poser des questions sur les personnes que l'on appelait, à l'instar de mon lointain parent, « guérisseurs », " panseurs», ou " rebouteux ». Mon intérêt pour ces personnes soignantes n'était donc pas le fruit du hasard, ni par conséquent ma recherche de thèse sur le pluralisme thérapeutique en Médoc. Adoptant une posture agnostique à leur égard, je me sentais à la fois proche et étrangère à leurs univers. Pour nombre de thérapeutes cependant, mon travail sur eux signifiait clairement que mon destin me guidait vers une activité thérapeutique non conventionnelle similaire à la leur.

Cela a été le cas avec Christine, qui développait elle-même ses capacités de guérison, et avec Laura, lors de la consultation précédemment évoquée. "Bon, on va pas tourner autour du pot 107 ans ! Tu sais ce que t'es ! », m'avait-elle affirmé au cours de la séance. Je n'avais rien répondu, attendant des explications, livrées avec un mélange d'ironie et de bienveillance : " Mademoiselle fait une thèse sur les guérisseurs! Tu sais ce que $t^{\prime}$ es !» Je restai silencieuse. "Tu sais ce que $t^{\prime}$ es, petite médium qui s'ignore ! Petite sorcière ! » J'acquiesçai et marmonnai un " oui, peut-être... », commençant à imaginer que le don de mon aïeul s'était peut-être transmis jusqu'à moi. "Ecoutant les anges » précédemment appelés (phénomène qu'elle appelait "clairsentience »14), la sorcière a ajouté : " On me dit que tes dons seraient plutôt médiumniques. Tu pourrais être une guideuse d'âmes. Ce sont ceux qui aident à faire passer les morts! Mais toi, ça va arriver dans la trentaine ». Puis se tournant vers le mur d'en face, elle s'est mis à «leur » parler : «Oui, j'ai compris qu'elle a quelque chose à faire parmi nous. Mais laissez-la finir sa thèse d'abord, s'il vous plaît ! ». Un peu interloquée, j'ai dit à Laura que je ne savais pas comment $\mathrm{m}^{\prime} \mathrm{y}$ prendre pour devenir guideuse d'âmes. Elle a répondu avec douceur : "Ne t'en fais pas. Ça va arriver tout seul et tu le sentiras. Il ne faudra pas avoir peur ».

Sur le moment, l'idée que cela puisse être vrai m'a à la fois fascinée et terrifiée. La medium avait fait sonner ces affirmations à la manière d'une « révélation » : alors que je ne $\mathrm{m}^{\prime} \mathrm{y}$ attendais pas, elle les avait énoncées comme une évidente vérité qui serait restée latente et cachée jusqu'alors, attendant le «bon moment» pour être exprimée. Mes émotions ont été amplifiées par l'effet de surprise qui s'ajoutait à l'ambiance déjà étrange de la consultation. Elles se sont aussi mêlées à ce moment-là à un sentiment de reconnaissance de mon histoire familiale, laquelle disait que le don de mon arrière-grand-père guérisseur s'était transmis à certains membres de la famille sans que l'on ne sache à qui ni comment. Je me demandais confusément si les mots de la medium signifiaient que c'était réel et que c'était moi. Concernant mon travail de thèse, cette séance $\mathrm{m}^{\prime}$ a rendue très enthousiaste car se concrétisait la possibilité de réaliser une ethnographie immersive des pratiques sorcellaires

14 Dans le milieu ésotérique, la clairsentience renvoie à une forte sensibilité aux ressentis et émotions des autres, aux ambiances d'un lieu, ainsi qu'à la présence et aux messages des personnes défuntes ou d'entités surnaturelles (comme les Anges ou les « êtres de Lumière »). 
féminines locales, par le biais de mon initiation et d'une expérience «de l'intérieur ».

L'ensemble de ces éléments permet de comprendre que la medium a mis en œuvre un processus d'assignation identitaire, certes surnaturelle, à laquelle elle pressentait une certaine adhésion de ma part, que ce soit sur le plan personnel ou professionnel. D'ailleurs, elle me dit plus tard qu'elle connaissait un peu le milieu de la recherche pour avoir eu des amies en thèse ; elle en avait déduit que les chercheurs choisissent rarement leur sujet de recherche au hasard. De mon côté, pour les raisons énoncées qui étaient propres au contexte particulier de ma vie à ce moment précis, j'ai accepté ce jour-là d'expérimenter pleinement l'univers proposé par cette femme. Ainsin le degré d'adhésion aux discours du medium semble très dépendant du moment de vie et des attentes du consultant ainsi que de la capacité du medium à en percevoir les enjeux et à en proposer une lecture interprétative satisfaisante. Mon degré d'adhésion a par la suite fluctué au fil du temps, des rencontres et des expériences.

$\mathrm{Au}$ cours de l'enquête, j'ai en effet clarifié ma posture personnelle et ethnographique en assumant un point de vue que l'on peut qualifier d'agnostique. Cette posture était à mon sens pertinente pour avoir accès et comprendre de l'intérieur les ressorts de l'initiation sorcellaire dans l'entre-soi féminin en Médoc, et saisir ce qui pouvait motiver ces femmes à s'adonner à la sorcellerie au quotidien. Par la suite, le travail d'écriture a nécessité que j'adopte une posture plus distanciée et réflexive, passant notamment par la mise en perspective de cette expérience particulière: il apparait qu'en acceptant, dans le cadre du travail de terrain, la possibilité d'être prédisposée à une activité médiumnique, j'ai rendu possible le fait de devenir l'apprentiesorcière de Christine et de Laura. Le rôle qui $\mathrm{m}^{\prime}$ a alors été attribué nécessitait que je suive sérieusement les enseignements des deux sorcières, désormais convaincues qu'elles pouvaient me transmettre leurs savoirs.

Précisant qu'elle pratiquait uniquement la bienfaitrice magie blanche, Christine me confia des «secrets de sorcière" (rituels de protection et de purification) et des "prières secrètes ». Je me confectionnais un pendule sur ses conseils et ceux de Laura et m'exerçais à le faire tournoyer tout en lui posant des questions. Un jour, interprétant la perte de son pendule habituel, Christine m'a annoncé que c'était le signe que le moment était venu de me transmettre son savoir. Très flattée d'être ainsi élue et de pouvoir approfondir mon apprentissage, j'ai été en même temps inquiète de mettre ma recherche en péril, comprenant que Christine souhaitait rapidement m'enseigner la communication médiumnique. Pour Deirdre Meintel, «la médiumnité concerne toute perception venant de $l^{\prime}$ " au-delà », une expression qui désigne le monde des esprits » mais aussi parfois celui des défunts (Meintel, 2011 : 91). Comme elle, j'ai été étonnée de voir que ce que je croyais être un «don » inné pouvait faire l'objet d'un apprentissage. Pour autant, je n'ai pas eu l'audace ni la confiance d'expérimenter autant qu'elle le lâcher-prise et la disponibilité 
mentale pour le domaine du ressenti15. Pour moi, cela participait d'une perte de contrôle que j'ai décidé de refuser à ce moment-là, ne souhaitant pas plus dévoiler, en déconstruisant tous mes filtres, mes ressentis et émotions. De plus, j'ai craint que cette initiation m'incite à passer un brutal cap de nonretour quant à ma façon d'être au monde, ce dont mes collègues se souciaient par ailleurs, à chaque fois que je leur annonçais mes intentions d'expériences de soins non conventionnels sur le terrain. Ils s'assuraient régulièrement que mon immersion ne m'empêche de revenir à mon statut de doctorante en ethnologie.

Percevant mes réticences, Christine semblait ne pas comprendre pourquoi je résistais à un pareil héritage. De mon côté, je n'osais d'abord pas lui dire que je restais très intéressée par son savoir et ses pratiques mais que je n'étais pas disposée à les faire miens pour l'instant, préférant maintenir une certaine distance pour mener à bien ma recherche. Je lui ai aussi confié ma peur plus personnelle du monde des anges et des défunts. Nous avons convenu que je resterais observatrice de ses pratiques ésotériques, tout en devenant son apprentie quant aux usages des plantes médicinales, perçus par elle comme un autre aspect de la sorcellerie contemporaine. Mon statut d'apprentiesorcière s'est donc le plus souvent volontairement limité à l'étude des remèdes empiriques populaires et à l'observation des pratiques magiques. N'abandonnant pas totalement l'envie d'expérimenter des techniques favorisant un lâcher-prise émotionnel, j'ai assisté à des cours de yoga et de méditation à Bordeaux et dans le Médoc ainsi qu'à un stage collectif de " découverte de l'intuition », tout en poursuivant mes recours aux soins chez les thérapeutes non conventionnels rencontrés. Je me suis aussi employée à la lecture de livres à succès dits "de développement personnel ", parfois conseillés par Christine, et qui entendaient offrir au lecteur la possibilité de " travailler sur soi », empruntant ce terme aux champs de la psychologie, afin d'apprendre à maîtriser ses émotions et à dépasser ses propres modes de pensée et d'agir lorsqu'ils sont vécus comme empêchants ou potentiellement nocifs pour la santé (Marquis, 2014).

Ces lectures revêtaient un double intérêt: d'une part, elles constituaient pour moi une source de données ethnographiques me permettant de comprendre l'importance de cette littérature dans la diffusion des principes

15 Deidre Meintel explique que «le cheminement dans la médiumnité (...) contraste de manière frappante avec celui de l'apprentissage académique où, généralement, le savoir est acquis par un processus d'appropriation de connaissances. Par un effort intellectuel soutenu, on finit par 'maîtriser' un domaine, un phénomène. Sur le terrain, habituellement, le chercheur suit une démarche similaire (...). Entrer dans l'expérience de la voyance (...) nous oblige à apprendre autrement que par le travail académique. (...) Dans le groupe, les élèves 'désapprennent' la censure des impressions qui passeraient normalement sous silence. (...) le médium doit faire abstraction de toute prétention au contrôle. Il faut plutôt adopter une attitude de foi (s'ouvrir à la possibilité de la voyance malgré nos présuppositions au sujet de nos capacités) et d'humilité (accepter la possibilité de ne rien recevoir (...). » (Meintel, 2011 : 103). 
des soins non conventionnels, et d'autre part, elles ont pu alimenter ma propre quête identitaire et spirituelle. Cette porosité qui a pu avoir lieu entre travail de terrain et expérience personnelle ne doit pas être comprise comme un frein à l'analyse mais comme une opportunité d'observer, a posteriori et par le biais d'un travail de réflexivité, les effets intérieurs et intimes de ces pratiques de soins. J'ajouterai que ma directrice de thèse et mes collègues ont joué un rôle important dans ce travail de réflexivité, en me permettant de leur raconter régulièrement les expériences vécues sur le terrain ou en lien avec lui. Le travail de mise en récit du vécu réalisé avec ces personnes $\mathrm{m}^{\prime}$ aidait à prendre de la distance par rapport au terrain et à mieux en saisir les logiques internes dans lesquelles j'étais parfois impliquée.

La démarche choisie m'a permis d'affiner mes questionnements et de comprendre ce que Christine et Laura entendaient par lâcher-prise et «devenir sorcière » au-delà de la seule maîtrise de pratiques ésotériques comme on pourrait simplement le supposer. En effet, le travail d'écoute et d'interprétation des émotions, même les plus sensibles, peut être envisagé comme un levier possible pour assoir pleinement leur subjectivité, et particulièrement en tant que femme. Devenir une sorcière comme elles, signifiait aussi apprendre à faire fi des normes (de pudeur, de genre) qui régulent l'expression des émotions, en construisant comme un véritable pouvoir la capacité à assumer et dire ses ressentis.

\section{Vers une redéfinition de soi en tant que femme}

Lors de notre première rencontre, Laura m'avait expliqué qu'elle révélait régulièrement aux femmes, quand elle en avait l'intuition, leur potentiel de «sorcière ", comme elle l'avait fait pour Christine, puis pour moi. Grace aux entretiens et observations menés auprès d'elles, j'ai pu saisir que la sorcière, loin d'être un être maléfique, représentait une femme ayant choisi d'échapper à la domination masculine, menant une existence marginale du fait de ses choix. La figure de la sorcière est connue pour ses compétences magiques et connaissances en matière de guérison, mais aussi pour son dévoiement aux rôles féminins socialement attendus (Michelet, 1862(1966), Verdier, 1979; Planté, 2002). Ces conceptions se retrouvaient dans les pratiques thérapeutiques de Laura vouées aux maux de femmes : elle avait par exemple développé une représentation de la physiologie féminine et de ses cycles inspirés de la médecine humorale. Invitant les femmes à expérimenter différemment leurs menstruations au fil des semaines et des symboles associés, elle était convaincue de l'importance de se défaire du tabou des règles au niveau individuel dans la construction de soi, mais aussi au niveau social, dans l'élaboration d'une forme de fierté féminine partagée et reliée avec la nature. C'est ainsi qu'au cours d'une autre séance thérapeutique réalisée avec elle, elle insistant sur la célébration du "sacré féminin », valorisé dans le mouvement New Age au travers de symboles tels que la PachaMama ou Gaïa (Rocchi, 2000 ; Bishop, 2004 ; Grisoni, 2011). Chez Laura, d'autres figures 
mythiques étaient mobilisées au prisme d'une interprétation féministe: Eve par exemple, n'étant pas considérée comme la première pécheresse, mais comme ayant courageusement goûté au fruit de la connaissance puis engendré avec Adam toute l'humanité ; Lilith la première femme d'Adam et son égale, se serait séparée de lui à cause de ses désirs sexuels incessants et de sa domination physique pendant le coït.

L'engagement de la thérapeute, le cheminement de Christine et les savoirs qu'elles m'ont transmis permettent de comprendre leur démarche consistant en la prise de conscience de leurs émotions négatives, puis de leur mise en perspective avec les normes, notamment de genre, afin de mieux saisir l'influence de leur histoire et du social dans leurs ressentis et d'en transcender les souffrances vécues. Pour ces femmes au mode de vie quelque peu marginal, cette quête constante de sens et de bien-être s'exerçait au quotidien comme une activité donnant un véritable but à leur existence, et redéfinissant leur identité en tant que femmes, dans leur rapport à soi et aux autres.

En ne me plongeant pas totalement dans cette démarche, j'ai finalement suivi un cheminement comparable à de nombreuses femmes usagères de soins non conventionnels, qui incarnent alors plus ponctuellement et parfois à leur insu cette vision féministe de la "sorcière ». Le travail émotionnel engagé avec les thérapeutes ou en autonomie amène à une perspective phénoménologique de l'expérience d'être femme, incitant chacune à se penser en tant que sujet et à choisir d'incarner ou non les rôles construits comme féminins. Si ce souci de soi est d'abord pensé comme une condition de bien-être individuel, il semble aussi participer à renouveler le féminisme contemporain, tel que le propose la politiste Camille Froidevaux-Metterie (2015), qui plaide pour une revalorisation et une reconnaissance de leurs spécificités par les femmes ellesmêmes.

\section{CONCLUSION}

Dans cet article, l'approche par une anthropologie réflexive des émotions permet de rendre compte des différents ressentis éprouvés selon que j'ai été la confidente, l'usagère de soins non conventionnels ou l'apprentie-sorcière de mes interlocutrices. Cette démarche n'entend pas seulement illustrer les difficultés d'accès au domaine des maux de femmes ou à celui des soins non conventionnels, mais aussi donner à voir l'élaboration de mon expérience ethnographique dans ces contextes précis comme une source de données susceptibles d'en alimenter la compréhension. L'analyse de mon vécu montre que les affects - tantôt voilés, tantôt dévoilés - ont occupés une large place dans mes interactions avec les femmes dès lors qu'elles avaient lieu dans les contextes localement propres à la confidence féminine et à la sorcellerie.

Questionner et recueillir la parole sur les maux de femmes m'a amenée à faire dire l'indicible à mes enquêtées et à porter ensuite le poids de charges 
émotionnelles lourdes, montrant combien la pudeur et le tabou véhiculés par les normes de genre participent tant à la production qu'à la dissimulation des souffrances des femmes. La place centrale des émotions dans les soins non conventionnels a été abordée au prisme de la sociologie des religions (Champion, Hervieu-Léger, 1990; Rocchi, 2000) mais rarement par l'anthropologie de la santé : or mon expérience de recours à ces pratiques thérapeutiques $\mathrm{m}$ 'a permis de comprendre le rôle joué par les praticiens dans le dévoilement d'émotions négatives autrement masquées. Grâce aux méthodes plus classiques de l'enquête ethnographique (entretiens, observations), j'ai constaté que le dévoilement des émotions des patientes constituait à la fois un support diagnostique pour les praticiens et une forme de soulagement cathartique pour les usagères. Le fait de vivre ces recours $\mathrm{m}^{\prime} \mathrm{a}$ permis de saisir en quoi ces soins favorisent un sentiment apaisant de reconnaissance par l'autre de ses souffrances, ainsi que la sensation de pouvoir les extérioriser pour mieux agir sur elles. Le travail émotionnel proposé passe par l'acceptation résiliente des maux et par leur intégration au parcours biographique de chacun(e). Un sentiment de désamorçage des émotions négatives intervient notamment à mesure que l'on change son regard sur les situations douloureuses. J'ai en effet vécu ces soins, puis mon statut d'apprentie-sorcière, comme un apprentissage visant à modifier ma perception du monde, de la place et du rôle que j'y occupe, de mon esprit et de mon corps, tout en continuant mes activités et relations sociales habituelles. La phrase que m'a dit une professeure de yoga rencontrée dans le Médoc résume cette sensation : «En fait, tu restes toi... ou tu redeviens toi. Rien de ce qui t'entoure n'est différent et pourtant tout a changé ». La mise en perspective des souffrances de femmes avec ces soins et avec l'initiation au " devenir sorcière », a fait apparaitre, chez moi comme certaines enquêtées, un processus individuel de transformation de la façon de se penser et d'être au monde, guidé par des représentations sociales du féminin particulièrement valorisantes et intimement perçues comme émancipatrices en regard des normes de genre.

\section{BIBLIOGRAPHIE}

Elsa BISHOP, "Constructions imaginaires de l'espace californien au sein du New Age", Matériaux pour l'histoire de notre temps, $\mathrm{n}^{\circ} 75,2004$, pp. 28-38. URL: www.persee.fr/doc/mat_0769-3206_2004_num_75_1_991

Rémi BORDES (dir.), Dire les maux: anthropologie de la parole dans les médecines traditionnelles, Paris, L'Harmattan, 2011.

Carole H. BROWNER, Carolyn F. SARGENT, Reproduction, globalization, and the state: New theoretical and ethnographic perspectives, Duke University Press, 2011.

CAMBRELLA, Project of the pan-European research network for complementary and alternative medicine (CAM) URL: http:/ / cambrella.eu/home.php 
Sarah CANT, Ursula SHARMA, A New Medical Pluralism: complementary medicine, doctors, patients and the state, Routledge, 1999.

Françoise CHAMPION, Danièle HERVIEU-LEGER (dir.), De l'émotion en religion, Paris, Éds du Centurion, 1990.

Patrice COHEN, Ilario ROSSI, Aline SARRADON, Olivier SCHMITZ, « Des systèmes pluriels de recours non conventionnels des personnes atteintes de cancer: Une approche socioanthropologique comparative (France, Belgique, Suisse) », Rapport INCA, 2010.

Patrice COHEN, Ilario ROSSI, "Le pluralisme thérapeutique en mouvement», Anthropologie $\mathcal{E}$ Santé, $\mathrm{n}^{\circ} 2,2011$ [En ligne]. URL: http://anthropologiesante.revues.org/606

Mary DOUGLAS, De la souillure, Paris, Maspéro, 1971.

Marcel DRULHE, «Émotion et Société : un enjeu sociologique », Face à face, no 9, 2006, pp. 12-17.

Marcel DRULHE, « Le travail émotionnel dans la relation soignante professionnelle. Un point de vue au carrefour du travail infirmier », Sciences sociales et santé, volume 21, no 1, 2000, pp. 15-29.

Nicole DURISCH-GAUTHIER, "Le marché de la guérison », in Nicole DURISCHGAUTHIER, Ilario ROSSI, Jörg STOLZ, Quêtes de santé. Entre soins médicaux et guérison spirituelles, Genève, Éditions Labor et Fides, 2007, pp.37-55.

Jeanne FAVRET-SAADA, Désorceler, Paris, Ed. de L'Olivier, 2009.

Jeanne FAVRET SAADA, Les mots, la mort, les sorts, Gallimard, Paris, 1977.

Jeanne FAVRET SAADA et Josée CONTRERAS, «Ah! La féline, la sale voisine ! , Terrain, n $^{\circ} 14,1990$, pp 20-31.

Fabrice FERNANDEZ, Samuel LEZE et Hélène MARCHE (dir.), Le langage social des émotions. Etudes sur les rapports au corps et à la santé, Parsi, Économica, 2008.

Camille FROIDEVEAUX-METTERIE, La révolution du féminin, Paris, Gallimard, 2015.

Ghislaine GALLENGA, "L'empathie inversée au cœur de la relation ethnographique », Journal des anthropologues, $\mathrm{n}^{\circ} 114-115,2008$ [En ligne]. URL: http://jda.revues.org/319.

Christian GHASARIAN, "Santé alternative et New Age à San Francisco », in Jean BENOIST et Raymond MASSE (dir.), Convocations thérapeutiques du sacré, 2002, pp. 143-163.

Anahita GRISONI, Sous les pavés, la terre: culte du bien-être et nouveaux métiers : la naturopathie en transformation à la conquête du marché, Thèse de doctorat de sociologie, Paris, EHESS, 2011.

Laurence KOTOBI et al., "Santé reproductive dans le Médoc (France) : femmes, précarité, migration », Rapport final de la recherche : Inégalités d'accès aux soins en santé reproductive et génésique des femmes "précaires » et/ou "migrantes » du Médoc, IRESP/INSERM, 2010-2013 (Resp. L. Kotobi), Université de Bordeaux, Décembre 2013. Dact.

Laurence KOTOBI, « Réflexions sur l'altérité migrante comme support de traitements différentiels à l'hôpital et sur la production des données par l'ethnologue. », in Chantal CRENN et Laurence KOTOBI (dir.), L'ethnicité en débats : pratiques françaises, Paris, Armand Colin, 2012, pp. 179-229. 
David LE BRETON, «La construction sociale de l'émotion», Les Nouvelles d'Archimède, 2004, n³5, pp. 4-5.

Clara LEMONNIER, Quêtes de soins au féminin. Une ethnographie des "maux de femmes " et du pluralisme thérapeutique en Médoc (France), Thèse de doctorat en anthropologie, Université de Bordeaux, 2016.

Clara LEMONNIER, Ritualiser l'avortement pour faire passer la pilule, Enquête sur l'Interruption Volontaire de Grossesse dans le centre d'orthogénie d'un hôpital aquitain, Mémoire de Master 1 en anthropologie, Université Bordeaux 2, 2010.

Aurélia MARDON, «Honte et dégoût dans la fabrication du féminin. L'apparition des menstrues », Ethnologie française, vol. 41, n¹, 2011, pp. 33-40 [En ligne]. URL: www.cairn.info/revue-ethnologie-francaise-2011-1-page-33.htm

Nicolas MARQUIS, Du bien-être au marché du malaise. La société du développement personnel, Paris, PUF, 2014.

Marcel MAUSS, "L'expression obligatoire des sentiments (rituels oraux funéraires australiens)», Journal de psychologie, $\mathrm{n}^{\circ} 18,1921 . \quad \mathrm{URL}$ : http://classiques.uqac.ca/classiques/mauss_marcel/essais_de_socio/T3_expression _sentiments/expression_sentiments.html

Deirdre MEINTEL, «Apprendre et désapprendre: Quand la médiumnité croise l'anthropologie », Anthropologie et sociétés, vol. 35, no 3, 2011, pp. 89-106.

Dominique MEMMI, "Administrer une matière sensible, Conduites raisonnables et pédagogie par corps autour de la naissance et de la mort », in Didier FASSIN et Dominique MEMMI (dir.), Le gouvernement des corps, Paris, EHESS, 2004, pp.135-153.

Catherine MERCADIER, Le travail émotionnel des soignants à l'hôpital. Le corps au cour de l'interaction soignant soigné, Paris, Ed. Seli Arlan, 2002.

MIVILUDES, Guide-Santé et dérives sectaires, Paris, La documentation française, 2012 [En ligne]. URL: http://www.derivessectes.gouv.fr/sites/default/files/publications/francais/guide_sante_complet.pdf

OMS, «Stratégie de l'OMS pour la médecine traditionnelle pour 2002-2005 », 2002, Genève [En ligne]. URL : http://apps.who.int/medicinedocs/pdf/s2298f/s2298f.pdf

OMS, «Stratégie de l'OMS pour la médecine traditionnelle pour 2014-2013 », 2013, Genève [En ligne].. URL : http://apps.who.int/iris/bitstream/10665/95009/1/9789242506099_fre.pdf

Patricia PAPERMAN, «La question des émotions : du physique au social », L'Homme et la société, n 116, 1995, pp. 7-17.

Clémentine RAINEAU, "La pratique de l'hypnose, de la visualisation ou de l'autohypnose par des personnes atteintes d'un cancer : une transformation de soi ? ", Anthropologie E Santé, Revue internationale francophone d'anthropologie de la santé, $\mathrm{n}^{\circ} 2$, 2011, [En ligne]. URL : https:/ / anthropologiesante.revues.org/ 666

Clémentine RAINEAU, Maladie et infortune dans l'Auvergne d'aujourd'hui : médecins, guérisseurs et malades: d'un village montagnard à l'hôpital, Thèse de doctorat en anthropologie, Paris, EHESS, 2001.

Paul RICOEUR, «La souffrance n'est pas la douleur», Autrement. Série mutations, $\mathrm{n}^{\circ} 142,1994$, pp. 58-69.

Bernard RIMÉ, Le partage social des émotions, Paris, PUF, 2009. 
Isabelle ROBARD, Médeci`es ‘o`co`ve`tio`elles et droit: la `écessaire $i$ tégratio`da`s les systèmes de sa' té e` Fra`ce et e`Europe, Paris, Litec, 2002.

Valérie ROCCHI, "Du Nouvel Age aux réseaux psycho-mystiques", Eth ologie fra çaise, XXX, 4, 2000, pp. 583-590.

Ilario ROSSI, "La parole comme soin: cancer et pluralisme thérapeutique », A`thropologie $\mathcal{E} \quad S a^{`} t e ́, \quad n^{\circ} 2,2011$ [En ligne]. URL: http:/ / anthropologiesante.revues.org/659

Ilario ROSSI, "Soigner par la parole sacrée. Les défis réflexifs du chamanisme américain », $i$ Raymond MASSÉ et Jean BENOIST, Co`vocatio`s thérapeutiques du sacré, Paris, Karthala, 2002, pp. 327-345.

Juliette SAKOYAN, Sandrine MUSSO et Stéphanie MULOT, "Quand la santé et les médecines circulent. Introduction au dossier thématique Médecines, mobilités et globalisation ", A thropologie $\mathcal{E}$ Sa té, $\mathrm{n}^{\circ} 3,2011$ [En ligne]. URL : https:/ / anthropologiesante.revues.org/819

Olivier SCHMITZ, Les Médeci`es è parallèle, Multiplicité des recours au soi`e`Occide`t, Paris, Karthala, 2006.

RÉSUMÉ : Cet article s'intéresse au jeu de voilement et de dévoilement des émotions à divers moments des itinéraires thérapeutiques des femmes rencontrées dans une région rurale française, et recourant à des soins non conventionnels pour des problématiques de santé perçues comme spécifiquement féminines. Il examine successivement le vécu des souffrances de femmes et de leur tabou, la place centrale des émotions dans les soins non conventionnels auxquels certaines recourent et les effets du travail émotionnel de celles qui s'engagent dans une quête de sens et d'identité, supportée par ce type de soins. Une réflexion méthodologique est apportée concernant l'étude de ces domaines peu visibles, que sont la santé des femmes, souvent marquée par l'intime et le tabou, ou celui des pratiques sorcellaires et psycho-mystiques, souvent tenues au secret. L'approche développée considère l'expérience émotionnelle de la chercheuse sur son terrain comme pertinente pour comprendre les expériences et les subjectivités des femmes recourant à un pluralisme thérapeutique.

MOTS-CLÉS : émotions, affecté, réflexivité, santé reproductive, tabou, secret, femmes, soins non conventionnels, sorcellerie, ruralité, France. 